\title{
TEM Characterization of Ball Milled CIS and CIGS Nanoparticles.
}

\author{
I. I. Santana-García ${ }^{1}$, C. Kisielowski ${ }^{2}$ and H. A. Calderon ${ }^{1}$ \\ 1. Departamento de Física, ESFM-IPN, México D.F. 07338, México. \\ 2. Molecular Foundry and JCAP, LBNL, One Cyclotron Road, Berkeley, CA 94720, USA.
}

CIGS (CuInxGa $\mathrm{Ca}_{(1-\mathrm{x})} \mathrm{Se}_{2}$ ) is a semiconducting photovoltaic material with the chalcopyrite crystal structure and a band gap varying with $\mathrm{x}$ from approximately $1.0 \mathrm{eV}$ (CIS) to around $1.7 \mathrm{eV}$ (CGS). These nanoparticles can be used to transform solar energy into electricity and are attractive for solar cells. In this investigation reactive mechanical milling is used for synthesis. This is a simple procedure where a chemical reduction is induced during milling at room temperature. It gives rise to a fine nanoparticle distribution if coagulation is restrained e.g. by adding a dispersing media. The nanoparticles in the present report have all been produced by reactive milling $(\mathrm{x}=0.3)$, starting with chlorides and chemically reducing them with $\mathrm{Na}$ in an excess of $\mathrm{NaCl}$ to promote dispersion.

Figure 1 shows the chemical distribution of CIS particles via EDS mappings. After $10 \mathrm{~h}$ of reactive milling, nanoparticles acquire a homogeneous distribution of the involved chemical elements. Similar results are determined for CIGS nanoparticles. Shorter milling times have shown clear chemical inhomogeneities in the nanoparticles compositions especially in the case of Ga containing materials. Figure 1 shows agglomerated nanoparticles at a relatively low magnification. The average particle size as determined by diffraction techniques varies from 5 to $7 \mathrm{~nm}$ but earlier TEM observation also shows that many smaller particles can be found.

These semiconducting nanoparticles are rather beam sensitive. Figure 2 shows phase images of CIS nanoparticles and the changes that induce the beam interaction as a function of electron dose rate. In all cases, 40 experimental images are taken for the exit wave reconstruction procedure i.e., the particle is exposed for approximately $2 \mathrm{~min}$ [1]. As shown only rather low dose rates (lower than $60 \mathrm{e}^{-} / \AA \mathrm{s}$, can be used to preserve the as-synthesized condition of the nanoparticles. Higher dose rates produce unexpected changes in the agglomerates. Figure 3 shows phase images of CIS nanoparticles at a dose rate lower than the above determined limit. Different sizes are imaged, independent nanoparticles can be as small as 2 $\mathrm{nm}$. Larger particles normally agglomerate and appear as crystalline domains in the phase images. The chalcopyrite structure can be readily identified in the atomic column distribution in Fig. 3 together with distinctively brighter columns that most likely represent heavier atoms. Figure 4 shows phase images of CIGS nanoparticles. In all cases, the electron dose rate is lower than $30 \mathrm{e}^{-} / \AA$ s since the presence of Ga increases dramatically the sample beam sensitivity and the corresponding bean induced changes. Particles have sizes that range between 2 and $5 \mathrm{~nm}$ and often their shape is irregular. When observed in detail and along a low index zone axis, the chalcopyrite structure can be readily recognized (see Fig. 4c). This investigation is complemented by simulation and modeling.

References:

[1] H.A. Calderon, C. Kisielowski, P. Specht, B. Barton, F. Godinez-Salomon, O. Solorza-Feria. Micron 68 (2014), p. 164.

[2] Electron Microscopy was performed at the Molecular Foundry, which is supported by the Office of Science, Office of Basic Energy Sciences of the U.S. Department of Energy under Contract No.DEAC02-05CH11231. The research is partially supported by CONACYT (Proyecto FOINST. 75/2012, 148304 and 129207), IPN (COFAA, SIP). 


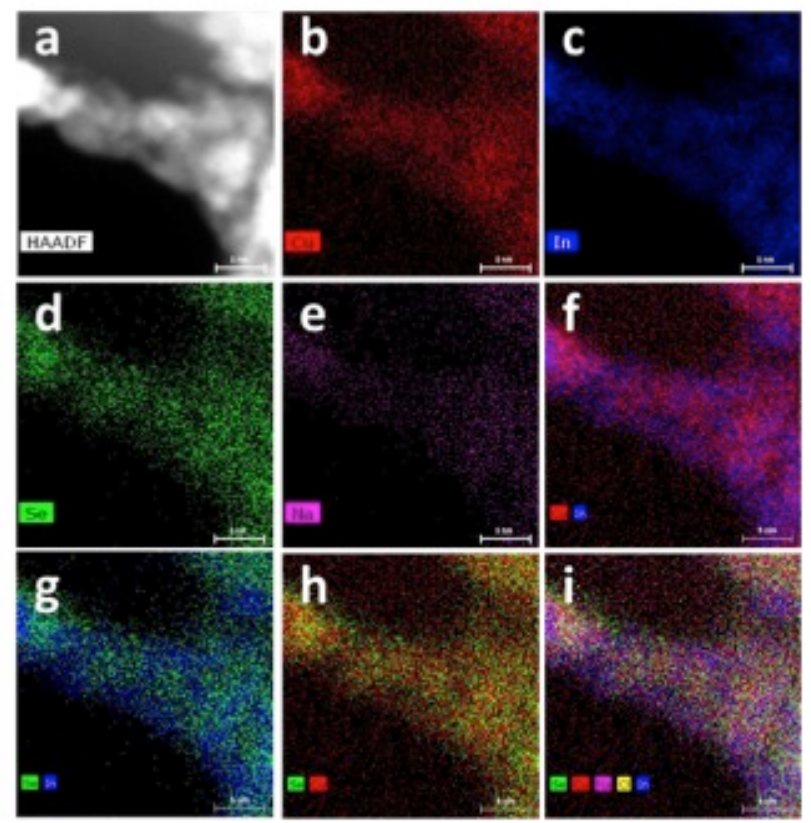

Figure 1. EDS Mappings of CIS nanoparticles (a) HAADF image, (b) $\mathrm{Cu}$, (c) In, (d) Se (e) Na, (f) $\mathrm{Cu}-\mathrm{In},(\mathrm{g}) \mathrm{Se}-\mathrm{In},(\mathrm{h}) \mathrm{Se}-\mathrm{Cu}$, (i) and $\mathrm{Cu}-\mathrm{In}-\mathrm{Se}$.

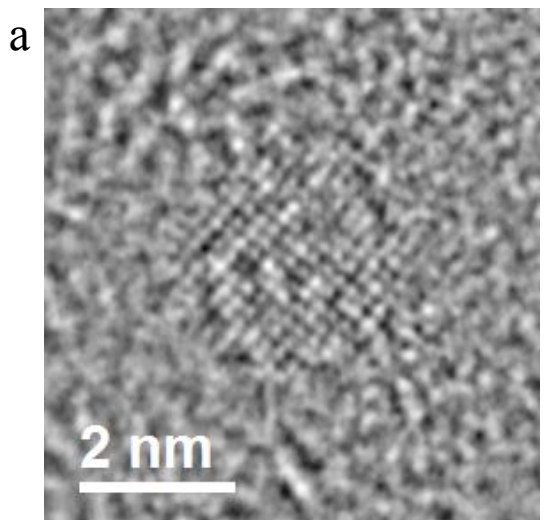

a
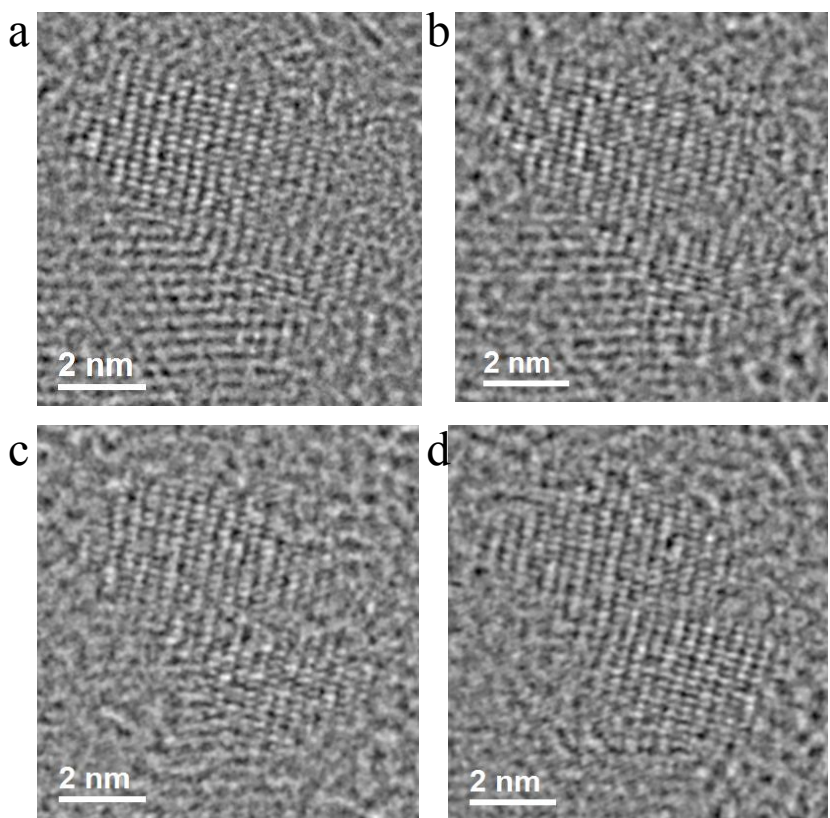

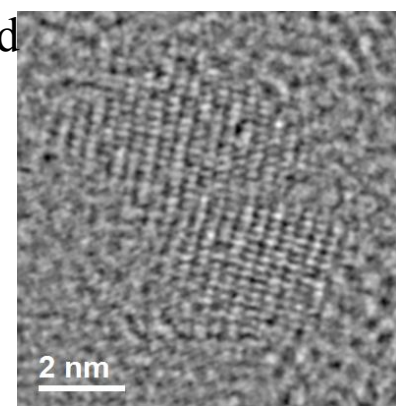

Figure 2. Phase images of CIS nanoparticles at different dose rate for approximately $2 \mathrm{~min}$. (a) 25 , (b) 58, (c) 400, (d) $1400 \mathrm{e}^{-/} \AA \mathrm{s}$.
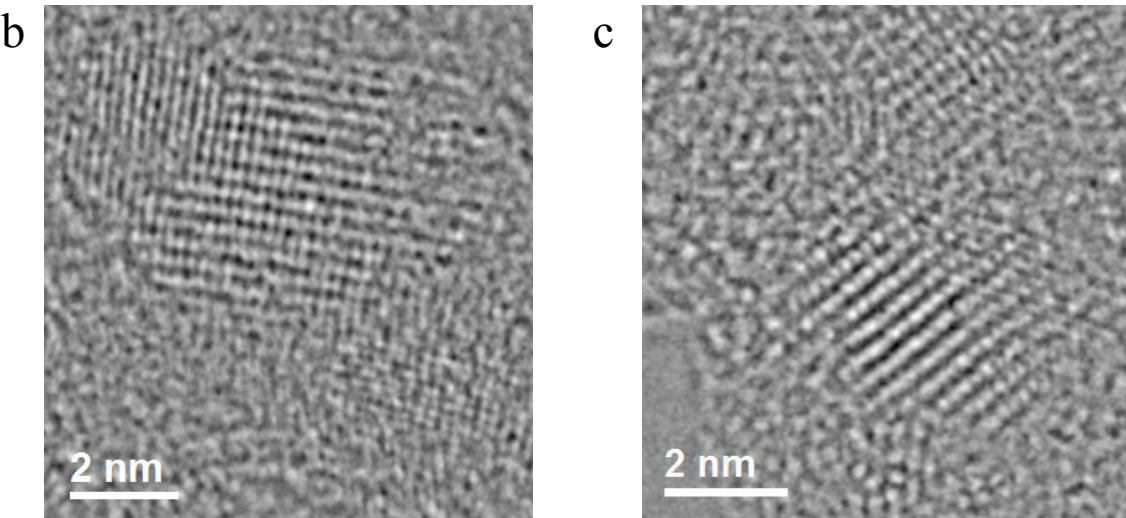

Figure 3. Low dose phase images of CIS nanoparticles after a EWR procedure. The expected chalcopyrite crystalline structure coincides with the observed atomic column distribution.

a

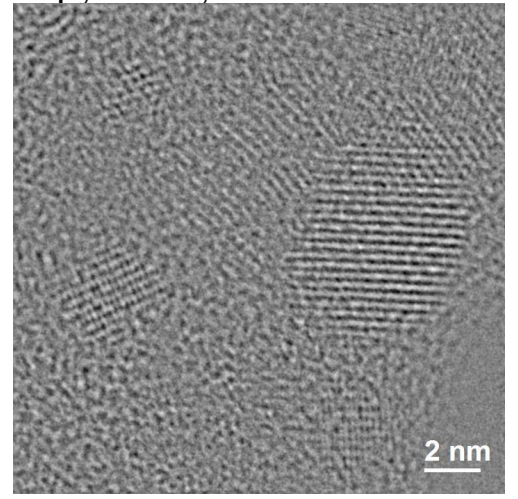

$\mathrm{b}$

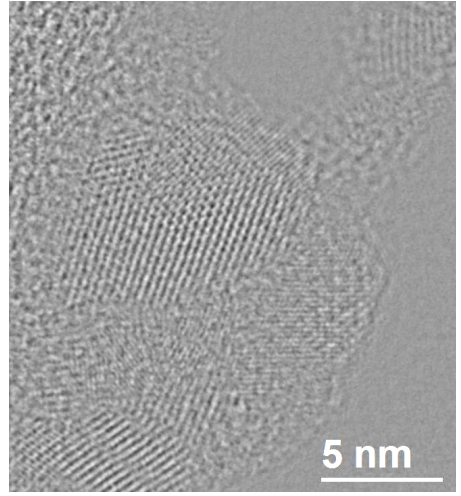

$\mathrm{c}$

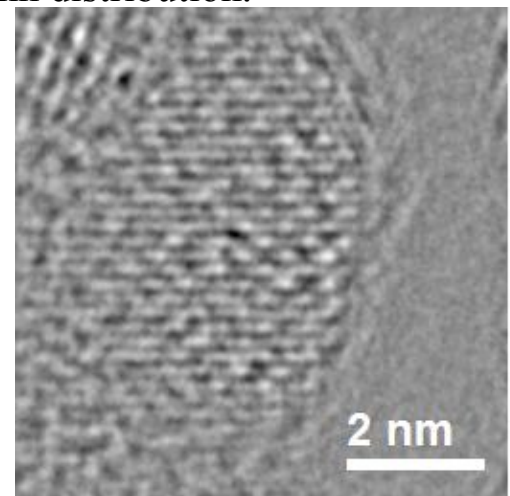

Figure 4. Low dose phase images of CIGS nanoparticles after an EWR procedure. The expected chalcopyrite crystalline structure coincides with the observed atomic column distribution. 